\title{
Platelet Rich Fibrin in Implant Dentistry: An Update
}

\section{Priyanka Rathod ${ }^{1}$,Siddhesh Borse ${ }^{1}$, Chetan Kakade ${ }^{1}$ and Sachin Chaware $^{2 *}$}

${ }^{1}$ Post-Graduate Student, Department of Prosthodontics, MGV Dental College, Nashik, India

${ }^{2}$ Ex-Professor, Department of Prosthodontics, MGV Dental College, Nashik, India

*Corresponding Author: Sachin Chaware, Ex-Professor, Department of Prosthodontics, MGV Dental College, Nashik, India.
Received: July 29, 2021

Published: August 26, 2021

(C) All rights are reserved by Sachin Chaware., et al.

\begin{abstract}
Bone graft materials is used for surgical defect area as a scaffolding material. however, an introduction of platelet rich fibrin has many benefits than bone graft materials. The major advantage of PRF that it is used autogenous blood without external thrombin, therefore there is no immunogenic reaction. The following paper is describing the advance application of PRF either used alone or combination with other graft materials in peri-implant region.
\end{abstract}

Keywords: Bone Graft Materials; Platelet Rich Fibrin; Implant Dentistry

\section{Introduction}

Post-operative surgical defect healing is a prominent factor for prognostic wound healing. To fulfil the volume of the defect bioactive surgical additivities were used which regulate inflammation and increase the speed of healing process [1]. Bioactive surgical additivities has been widely studied in implant dentistry, including various kind of bone graft materials with precise surgical technique. PRF was first introduced in 2000 by Joseph Choukroun and colleagues [2]. First introduced by Whitman., et al. in 1997. The second generation was developed by Dohan., et al. in 2001. The benefit of PRF that it stimulate the mitogenic response in the bone periosteum during normal wound healing for repair of the bone [3]. Apart from Surgical defect additives it has several outcome in regeneration of tissue for advancement in implant therapy.

\section{Preparation of protein rich fibrin}

The PRF preparation protocol is very simple and armamentarium required is same as that of PRP. Around $5 \mathrm{ml}$ of whole venous blood is collected in each of the two sterile vacutainer tubes of 6 $\mathrm{ml}$ capacity without anticoagulant. The vacutainer tubes are then placed in a centrifugal machine at 3000 revolutions per minute (rpm) for 10 minutes, after which it settles into the following layers: red lower fraction containing red blood cells, upper straw coloured cellular plasma and the middle fraction containing the fibrin clot. The upper straw coloured layer is then removed and middle fraction is collected, $2 \mathrm{~mm}$ below lower dividing line, which is the PRF. The mechanism which is followed here is that, fibrinogen which is initially concentrated in the high part of the tube, combines with the circulating thrombin due to centrifugation, to form fibrin. A fibrin clot is then obtained in the middle of the tube. Anticoagulants 
or enzymatic supplements may be required to separate PRP and platelet-poor plasma (PPP) the former with enough platelets for therapeutic use, yet less abundant than PPP at roughly $25 \%$ and $75 \%$ of the supernatant volume, respectively.

PRF activates neutrophil, fibroblast and macrophage, thereby it stimulate mesenchymal cells. Furthermore, it enhances cellular differentiation, matrix formation and collagen synthesis, and fibroblast activation. In addition, promote epithelial cell replication and growth factor proliferation [2].

\section{Application}

PRF membrane application is simplified and efficient technique with minimum blood manipulation as it is obtained by autogenous blood sample. Since, it is natural fibrin framework it does not require external thrombin and it has concentrated growth factor for longer duration tissue generation. It can be uses alone or combination with bone graft. Following are the advanced application of PRF membrane [1].

Biomaterial template with silk fibroin powder has been investigated for the restoration of peri-implant defects when mixed with Choukroun platelet-rich fibrin (PRF). The removal torque for the experimental group was $30.345 .06 \mathrm{Ncm}$, whereas it was 21.86 $3.39 \mathrm{Ncm}$ for the control. The difference between the 2 groups was statistically significant $(\mathrm{p}<01)$. Mean new bone formation was $51.9327 .90 \%$ in the experimental group and $11.6715 .12 \%$ in the control (P .003). Similarly, mean bone-to implant contact was 43.07 $21.96 \%$ in the experimental group and $15.3723 .84 \%$ in the control (P .002). A peri-implant defect can be successfully repaired by the application of Choukroun PRF and silk fibroin powder [4].

The difference sinus augmentation using a crestal approach in combination with an autologous leukocyte- and platelet-rich fibrin (PRF) concentrate. The mean residual subantral bone height of the alveolar ridge was $6.6 \mathrm{~mm}$ (range, 4 - $8 \mathrm{~mm}$ ). The mean increase in the height of implant sites by OMSFE/PRF was $3.4 \mathrm{~mm}$ (range, 2.5 - $5 \mathrm{~mm}$ ). A variety of 8- to $11.5-\mathrm{mm}$ long (mean length, $10.1 \mathrm{~mm}$ ) and 3.5- to 6-mm wide (mean width, $4.4 \mathrm{~mm}$ ) screw-type implants were used. Of the 138 implants that had been placed, 97 have been restored and in function for an average loading time of 5.2 months (range, 1 - 11 months). The mean healing time for the loaded implants was 4 months until abutment insertion (range, 3 - 5 months).
Three implants failed before loading for an early survival rate of both loaded and unloaded implants of 97.8\%. Conclusions: Early review of the OMSFE/PRF technique presented for localized sinus floor elevation and implant placement demonstrates a high degree of safety and success at sites with 5- to 8-mm residual subantral bone height [5].

In contrast to above method combination of leukocyte- and platelet-rich fibrin (L-PRF, Choukroun's technique) as sole subsinus filling material has been investigated. Study finding reported that stable implant healing. No implant was lost during this 6-year experience, and the vertical bone gain was always substantial, between 8.5 and $12 \mathrm{~mm}$ bone gain $(10.4,1.2)$. The final level of the new sinus floor was always in continuation with the implant apical end, and the peri-implant crestal bone height was stable. The use of L-PRF as sole filling material during simultaneous sinus-lift and implantation seems to be a reliable surgical option promoting natural bone regeneration [6].

Fibrin-rich blocks with concentrated growth factors (CGFs) alone as an alternative to graft material has been investigated and reported with no significant postoperative complications developed. New bone consolidation in all augmented maxillary sinus was observed along the implants on plain radiographs and on cone-beam computed tomograms. The success rate of implant was 98.2\% after an average of 10 months loading. Fibrin-rich blocks with CGFs act as an alternative to bone grafting and can be a predictable procedure for sinus augmentation [7].

The application of platelet-rich fibrin (PRF) into a peri-implant defect with histomorphometric analysis reported increase new bone formation and higher bone-to-implant contact with stable implant healing [8].

Marrelli M and Tatullo M (2013) [6] has assessed a predictable protocol of Platelet-Rich Fibrin (PRF) membrane in order to increase the peri-implant tissues maintenance around postextractive dental implants. Study findings observed the complete covering of the dental implants, with newly formed soft tissue of variable thickness between 1 and $3 \mathrm{~mm}$. Cortical bone adaptation from the time of implant placement up to 30 months following prosthetic restoration ranged from $0.4 \mathrm{~mm}$ to $1.7 \mathrm{~mm}$. The study showed a series of successful rehabilitations, with post-extraction 
implantology technique, in 99.8 percent of cases, despite the success rates in the medium and long-term post-extraction implantology reported in the literature range between 92.7 percent and 98.0 percent. Long-term maintenance of crestal bone and the rapid healing of soft tissue dimension with maintenance of peri-implant papilla were observed as outcomes after post-extractive implants insertion [9].

The difference bone regeneration among PRF-mixed Bio-Oss and Tisseel- mixed Bio-Oss in a canine sinus model. Six mongrel dogs were used in this study. After a 6 month healing period, bone formation in the graft sites and bone-implant contact were evaluated. The mean osseointegration rate was $43.512 .4 \%$ and new bone formation rate $41.85 .9 \%$ in the PRF/Bio-Oss composite sites. In contrast, the Tisseel/Bio-Oss composite sites they were 30.7, 7.9\% and $31.3,6.4 \%$. The findings from this study suggest that when platelet-rich fibrin is used as an adjunct to Bio-Oss particles for bone augmentation in the maxillary sinus, bone formation in the graft sites is significantly greater than Tisseel- mixed Bio-Oss [10].

One more evaluation of simultaneous sinus-lift and implantation with leukocyte- and platelet-rich fibrin (L-PRF, Choukroun's technique) as sole subsinus filling. The final level of the new sinus floor was always in continuation with the implant apical end, and the peri-implant crestal bone height was stable. The use of L-PRF as sole filling material during simultaneous sinus-lift and implantation seems to be a reliable surgical option promoting natural bone regeneration [11].

The effects of L-PRF and implant surface texture on bone healing around immediately placed implants reported a bone area fraction occupancy (BAFO) values were significantly higher for the Ossean relative to the dual acid-etched (DAE) surface on the larger mesial socket. The presence of L-PRF resulted in higher BAFO. The Ossean surface and L-PRF presence resulted in significantly higher BAFO. L-PRF and the micro/nanometer scale textured surface resulted in increased bone formation around immediately placed implant [12].

Similarly an experimental animal model with Histomorphometric analyses reported L-PRF-induced osseointegration and boneimplant contact (BIC) of the defects revealed that the L-PRF was detectable up to the second week. Application of L-PRF increased the rate and amount of new bone formation in the experimental group compared to the control group. Bone-to-implant contact was enhanced when the surface was pre-wetted with LPRF [13].

The peri-implant tissue changes around nano-pore implant surface with or without platelet rich fibrin (PRF) has been evaluated and reported the clinically, there was significant $(\mathrm{p}<0.001)$ increase in peri-implant probing depth (PIPD) from prosthetic phase to 9 months in both the groups and the increase was more in control group. However, the mean difference of change between the two groups was nonsignificant. Modified gingiva index (mGI) for Group II is significantly lower than that for Group I in prosthetic phase. Radiographically, in control group and experimental group, there was significant increase $(<0.01)$ in bone loss (BL) in mesial and distal aspect of implant from surgical to prosthetic phase, surgical to 9 months and from prosthetic phase to 9 months. There was greater BL in control group than in experimental group, in both mesial and distal aspect of implant, however the difference in BL was non-significant $(<0.01)$. There was greater BL in the distal aspect than in the mesial aspect in both the groups; however, the difference in BL was non-significant: PRF treatment may be a way to prevent bone loss during the surgical-to prosthetic phase [14].

The effect of autologous concentrated growth factor fibrin solution combined with Bio-Oss bone powder on mucosal healing and bone regeneration after oral implanted guided bone regeneration. There was no significant difference in the success rate of implants between the two groups ( $95.24 \%$ vs $97.56 \%$, P > 0.05). The complication rate of the experimental group was significantly lower than that of the control group $(2.38 \%$ vs $14.63 \%, \mathrm{P}<0.05)$. The mucosal color and swelling degree scores of the experimental group were significantly lower than those of the control group [C0.65 \pm $0.03)$ points vs $(2.01 \pm 0.15)$ points, $(1.10 \pm 0.37)$ points vs $(2.69 \pm$ 0.54 ) points, $\mathrm{P}<0.05]$, and the bleeding index, probing depth, and attachment loss were significantly lower than the control group $[(0.35 \pm 0.05)$ vs $(0.49 \pm 0.09),(3.39 \pm 0.62) \mathrm{mm}$ vs $(4.41 \pm 0.95)$ $\mathrm{mm},(3.02 \pm 0.66) \mathrm{mm}$ versus $(5.31 \pm 0.91) \mathrm{mm}, \mathrm{P}<0.05]$, bone graft height and osteogenesis height were significantly higher than the control group $[(2.61 \pm 0.50) \mathrm{mm}$ vs $(2.20 \pm 0.31) \mathrm{mm},(2.53 \pm$ $0.34) \mathrm{mm}$ vs $(2.02 \pm 0.27) \mathrm{mm}, \mathrm{P}<0.05]$. The degree of postoperative pain in the experimental group was significantly lower than that in the control group $(\mathrm{P}<0.05)$. Rich self-concentrating growth 
factor fibrin solution combined with Bio-Oss bone powder can effectively promote mucosal healing and bone regeneration after oral implant-guided bone regeneration and reduce postoperative pain and complications [15].

\section{Conclusion}

PRF membrane act as a physiologic glue which is either used alone or combination with other bone graft materials. It is used for periodontal defect, extraction socket healing in case of future implant placement, and in peri-implant area. it is completely noninvasive, with low risk surgical technique.

\section{Bibliography}

1. Borie E., et al. "Platelet-rich fibrin application in dentistry: a literature review". International Journal of Clinical and Experimental Medicine 8.5 (2015): 7922-7929.

2. Karimi K and Rockwell H. "The Benefits of Platelet-Rich Fibrin". Facial Plastic Surgery Clinics of North America - Journals 27.3 (2019): 331-340.

3. Naik B., et al. "Role of Platelet rich fibrin in wound healing: A critical review". Journal of Conservative Dentistry 16.4 (2013): 284-293.

4. Jang ES., et al. "Restoration of peri-implant defects in immediate implant installations by Choukroun platelet-rich fibrin and silk fibroin powder combination graft". Oral Surgery, Oral Medicine, Oral Pathology, Oral Radiology, and Endodontology 109.6 (2010): 831-836.

5. Toffler M., et al. "Osteotome-mediated sinus floor elevation using only platelet-rich fibrin: an early report on 110 patients". Journal of Implant Dentistry 19.5 (2010): 447-456.

6. Simonpieri A., et al. "Simultaneous sinus-lift and implantation using microthreaded implants and leukocyte- and platelet-rich fibrin as sole grafting material: a six-year experience". Implant Dentistry 20 (2011): 2-12.

7. Sohn DS., et al. "Bone regeneration in the maxillary sinus using an autologous fibrin-rich block with concentrated growth factors alone". Journal of Implant Dentistry 20.5 (2011): 389-395.
8. Lee JW., et al. "Restoration of a peri-implant defect by plateletrich fibrin". Oral Surgery, Oral Medicine, Oral Pathology, Oral Radiology, and Endodontology 113.4 (2012): 459-463.

9. Marrelli M and Tatullo M. "Influence of PRF in the healing of bone and gingival tissues. Clinical and histological evaluations". European Review for Medical and Pharmacological Sciences 17.14 (2013): 1958-1962.

10. Xuan F., et al. "A comparative study of the regenerative effect of sinus bone grafting with platelet-rich fibrin-mixed BioOss $\AA$ and commercial fibrin-mixed Bio-Oss $®$ : an experimental study". Journal of Cranio-Maxillofacial Surgery 42.4 (2014): e47-50.

11. Öncü E and Alaaddinoğlu EE. "The effect of platelet-rich fibrin on implant stability". The International Journal of Oral and Maxillofacial Implants 30.3 (2015): 578-582.

12. Neiva RF., et al. "The Synergistic Effect of Leukocyte PlateletRich Fibrin and Micrometer/Nanometer Surface Texturing on Bone Healing around Immediately Placed Implants: An Experimental Study in Dogs". BioMed Research International (2016): 9507342.

13. Öncü E., et al. "Positive effect of platelet rich fibrin on osseointegration". Medicina Oral, Patologia Oral, Cirugia Bucal 21.5 (2016): e601-607.

14. Khan ZA., et al. "Evaluation of peri-implant tissues around nanopore surface implants with or without platelet rich fibrin: a clinico-radiographic study". Biomedical Materials 13.2 (2018): 025002.

15. Lin Y., et al. "[Effect of autologous concentrated growth factor fibrin solution combined with Bio-Oss bone powder on mucosal healing and bone regeneration after oral implanted guided bone regeneration]". Shanghai Kou Qiang Yi Xue 29.3 (2020): 316-320.

\section{Volume 5 Issue 9 September 2021 (C) All rights are reserved by Sachin Chaware., et al.}

